\title{
Editorial
}

\section{Male Breast Cancer Patients: Lack of Evidence}

\author{
Isabell Witzel \\ Breast Center, Department of Gynecology, University Medical Center Hamburg, Hamburg, Germany
}

Although male breast cancer is a rare disease that accounts for less than $1 \%$ of all breast cancers, differences to female breast cancer in diagnosis and treatment are evident. The mean age at diagnosis is between 60 and 70 years; however, males of all ages can be affected with the disease. Primary standard treatment is a modified radical mastectomy with axillary intervention (sentinel node or axillary dissection). It is unknown how men perceive the body image change caused by the loss of one breast. Men are underrepresented in breast cancer trials, and most data on adjuvant treatment are based on evidence in women. The majority of male breast cancers are estrogen receptor positive. In this group, endocrine treatment with tamoxifen is recommended. Data supporting the use of an aromatase inhibitor in men with breast cancer are limited and even suggest higher mortality rates [1]. Results of the phase II clinical trial MALE of gonadotropin-releasing hormone analogue with tamoxifen or aromatase inhibitor in men are pending.

In addition to some uncertainty regarding the treatment of male breast cancer, articles in this issue of Breast Care make evident that another big concern for men with breast cancer are barriers in health care access. It can be assumed that these barriers as well as a lower disease awareness in men lead to a later diagnosis of male breast cancer with worse prognosis. Indeed, a recent study found higher mortality rates after cancer diagnosis among male patients with breast cancer compared with women [2].

In the focus of the current issue of Breast Care are psychosocial aspects, barriers in health care access, and genetic features of male breast cancer.

Nguyen et al. [3] highlight data on disease perception based on qualitative interviews with 18 men with breast cancer. In these interviews, men report being stigmatized by their surrounding family or friends of having a women's disease. Interestingly, men were rarely bothered by body image changes due to the mastectomy scar. Authors also explain coping strategies and report that an uncertainty about which doctor was responsible for treating male breast cancer existed in study participants.

To focus on the aspect of men's access to health care, Halbach et al. [4] conducted a study about male breast cancer patients' health care situation in Germany. One hundred male breast cancer patients answered a questionnaire, and 27 patients gave personal interviews. Results show that mostly primary care doctors were contacted when a lump in the male breast occurred. Authors identified fields of optimization for male breast cancer patient care, namely the need for tailored information material for men and standardization of providers of aftercare.

Pellini et al. [5] add data on family history of breast cancer and gene mutation status in a retrospective study of 69 men. Although $34.8 \%$ of the affected men had a positive family history of (female) breast cancer, only $11.8 \%$ were carriers of a pathogenic gene mutation (all BRCA2 mutation). There was no difference in the clinicopathological data of mutation carriers versus non-mutation carriers, but only 2 patients with a $B R C A 2$ mutation were available for this comparison. Authors also report results of genetic testing of healthy men of high-risk (breast cancer) families and found pathogenic BRCA1 or BRCA2 mutations in $50 \%$ of them. Authors conclude that surveillance programs would be established best in this cohort of healthy individuals. 
The presented articles of this focus make clear that studies of male breast cancer patients are challenging due to the low numbers of affected patients per center. However, the lack of evidence in this patient group has to be addressed by opening clinical trials also for male patients.

\section{Disclosure Statement}

The author declares that she has no conflicts of interest to disclose.

\section{References}

1 Eggemann $\mathrm{H}$, Ignatov A, Smith BJ, Altmann U, von Minckwitz G, Röhl FW, et al. Adjuvant therapy with tamoxifen compared to aromatase inhibitors for 257 male breast cancer patients. Breast Cancer Res Treat. 2013 Jan; 137(2):465-70.

2 Wang F, Shu X, Meszoely I, Pal T, Mayer IA, Yu Z, et al. Overall Mortality After Diagnosis of Breast Cancer in Men vs Women. JAMA Oncol. 2019 Sep;5(11):1589.
3 Nguyen TS, Bauer M, Maass N, Kaduszkiewicz $\mathrm{H}$. Living with Male Breast Cancer: A Qualitative Study of Men's Experiences and Care Needs. Breast Care (Basel). DOI: 10.1159/000501542.

4 Halbach SM, Midding E, Ernstmann N, Würstlein R, Weber R, Christmann S, et al. Male Breast Cancer Patients' Perspectives on Their Health Care Situation: A MixedMethods Study. Breast Care (Basel). DOI: 10.1159/000501956.
5 Pellini F, Granuzzo E, Urbani S, Mirandola S, Caldana M, Lombardi D, et al. Male Breast Cancer: Surgical and Genetic Features and a Multidisciplinary Management Strategy. Breast Care (Basel). DOI: 10.1159/000501711. 